MITSUBISHI ELECTRIC RESEARCH LABORATORIES

http://www.merl.com

\title{
Robust Dual Control MPC with Application to Soft-Landing Control
}

\author{
Cheng, Y.; Haghighat, S.; Di Cairano, S.
}

TR2015-064 July 2015

\begin{abstract}
Dual control frameworks for systems subject to uncertainties aim at simultaneously learning the unknown parameters while controlling the system dynamics. We propose a robust dual model predictive control algorithm for systems with bounded uncertainty with application to soft landing control. The algorithm exploits a robust control invariant set to guarantee constraint enforcement in spite of the uncertainty, and a constrained estimation algorithm to guarantee admissible parameter estimates. The impact of the control input on parameter learning is accounted for by including in the cost function a reference input, which is designed online to provide persistent excitation. The reference input design problem is non-convex, and here is solved by a sequence of relaxed convex problems. The results of the proposed method in a soft-landing control application in transportation systems are shown.
\end{abstract}

2015 American Control Conference (ACC)

\begin{abstract}
This work may not be copied or reproduced in whole or in part for any commercial purpose. Permission to copy in whole or in part without payment of fee is granted for nonprofit educational and research purposes provided that all such whole or partial copies include the following: a notice that such copying is by permission of Mitsubishi Electric Research Laboratories, Inc.; an acknowledgment of the authors and individual contributions to the work; and all applicable portions of the copyright notice. Copying, reproduction, or republishing for any other purpose shall require a license with payment of fee to Mitsubishi Electric Research Laboratories, Inc. All rights reserved.
\end{abstract}





\title{
Robust Dual Control MPC with Application to Soft-Landing Control
}

\author{
Y. Cheng, S. Haghighat, and S. Di Cairano,
}

\begin{abstract}
Dual control frameworks for systems subject to uncertainties aim at simultaneously learning THE unknown parameters while controlling the system dynamics. We propose a robust dual model predictive control algorithm for systems with bounded uncertainty with application to soft landing control. The algorithm exploits a robust control invariant set to guarantee constraint enforcement in spite of the uncertainty, and a constrained estimation algorithm to guarantee admissible parameter estimates. The impact of the control input on parameter learning is accounted for by including in the cost function a reference input, which is designed online to provide persistent excitation. The reference input design problem is non-convex, and here it solved by a sequence of relaxed convex problems. The results of the proposed method in a soft landing control application in transportation systems are shown.
\end{abstract}

\section{INTRODUCTION}

In many applications, offline parameter estimation and model identification is not possible due to numerous factors such as expensive process downtime, and parameters that change while in operation. Therefore, model (re)identification may need to be performed in real-time (online) and in closed-loop. However, simultaneous system identification and closed-loop control [1]-[7] is a challenging problem because of the conflicting objectives of control and identification, since control aims at stabilizing the system at the desired target, while to perform an effective identification, the system inputs have to (persistently) excite the system dynamics. The parameter estimate obtained by recursive least squares converges exponentially if the system is persistently excited [8]. However, a controller may interpret the excitation itself as a disturbance, thus rejecting it and canceling the persistent excitation.

In [4], an autoregressive system simultaneously identified an controlled with a finite horizon linear quadratic controller is shown to be stable if the information matrix increases within a number of steps. In [1], a model predictive control (MPC) and identification framework was proposed, where the system input is a sum of sinusoids with prescribed frequencies, for which the optimal amplitudes were proved to achieve persistent excitation. In [3] persistence of excitation over a $\Upsilon$-steps learning horizon is enforced from previous inputs and a single future output, requiring the solution of two convex quadratic programs. In [6] the MPC cost function is augmented with a 1-step learning objective, and in [9] set membership identification is applied with a MPC with robust constraint satisfaction.

Y. Cheng was an intern at Mitsubishi Electric Research Laboratories at the time of this research. email: yongfang09@gmail.com

S. Haghighat and S. Di Cairano are with Mitsubishi Electric Research Laboratories, Cambridge, MA, email: haghighat@merl.com, dicairanodieee.org
Along the lines of [7], in this paper we develop a robust dual control MPC (RDC-MPC), where however we only solve convex problems. The algorithm has general applicability although here we focus on its use for soft landing control [10], i.e., to precisely stop a moving object at a desired location, which is relevant in automotive, aerospace, and factory automation applications. To achieve a trade off between regulation and identification, a MPC algorithm with robust constraint satisfaction is modified by adding an additional (time-varying) term in the cost function, which quantifies the reduction of the parameter uncertainty. This results in an active learning method where the controller generates inputs that aim in part at regulating the system, and in part at exciting it to facilitate learning, where the trade off depends on the reliability of the current plant model. As opposed to the previous work [7], here we quantify reduction of the uncertainty due to an input sequence in terms of the predicted persistence of excitation measured through the change in the information matrix minimum eigenvalue over a learning horizon. To address the non-convexity of the problem, we propose a two-steps approach where both levels are executed online. First, an excitation input sequence is designed by solving a sequence of convex problems solving a rank minimization problem. Then, the excitation input sequence is used as reference input profile in an additional term of the MPC cost function, with a weight that depends on a function of the observed prediction error.

The paper is organized as follows. In Section II we briefly review polytopic linear difference inclusions, control invariant sets, and the soft landing control problem. Section III describes the two-steps RDC-MPC framework for pLDIs. The input design by convex problems is described in Section IV. In Section $\mathrm{V}$ the algorithm is applied to a soft landing problem related to the stop control of a transportation vehicle with uncertain parameters. The conclusions are drawn in Section VI.

Notation: $\mathbb{R}, \mathbb{R}_{0+}, \mathbb{R}_{+}$are the set of real, nonneative real, positive real numbers, and $\mathbb{Z}, \mathbb{Z}_{0+}, \mathbb{Z}_{+}$are the set of integer, nonneative integer, positive integer numbers. By $\oplus$ and co we denote Minkowski sum and convex hull, respectively. For a discrete-time signal $x$ with sampling period $T_{s}, x(t)$ is the state at sampling instant $t$, i.e., at time $T_{s} t$. By $[X]_{i, j}$ and $[x]_{i}$ we denote the $(i, j)$-th and $i$-th component of matrix $X$ and vector $x$, respectively.

\section{Preliminaries And Problem Definition}

In this section, we present some fundamental notions and preliminary results, and describe the dual control problem for soft landing control. 


\section{A. Polytopic Linear Difference Inclusions}

Given $\ell \in \mathbb{Z}_{+},\left\{\left(A_{i}, B_{i}\right)\right\}_{i=1}^{\ell}, A_{i} \in \mathbb{R}^{n_{x} \times n_{x}}, B_{i} \in$ $\mathbb{R}^{n_{x} \times n_{u}}, i=1, \ldots, \ell, B_{w} \in \mathbb{R}^{n_{x} \times n_{p}}$ and a polytope $\mathcal{W}=\operatorname{co}\left(\left\{w_{i}\right\}_{i=1}^{\eta}\right)$, where $w_{i} \in \mathbb{R}^{n_{p}}, i=1, \ldots, p$, the additive disturbance polytopic Linear Difference Inclusion (dpLDI) based on the $\ell$ vertex systems $x(k+1)=A_{i} x(k)+$ $B_{i} u(k), i=1, \ldots, \ell$ is

$$
x(k+1) \in \operatorname{co}\left\{A_{i} x(k)+B_{i} u(k)\right\}_{i=1}^{\ell} \oplus \operatorname{co}\left\{B_{w} w_{i}\right\}_{i=1}^{p} .
$$

The trajectories generated by (1) includes those of

$$
x(k+1)=\sum_{i=1}^{\ell}[\theta]_{i}\left(A_{i} x(k)+B_{i} u(k)\right)+\sum_{i=1}^{p}[\eta]_{i} B_{w} w_{i},
$$

where $\theta \in \mathbb{R}^{\ell}$ and $\eta \in \mathbb{R}^{p}$ are unknown and constant, $0 \leq$ $[\theta]_{i} \leq 1,0 \leq[\eta]_{i} \leq 1$, and $\sum_{i=1}^{\ell}[\theta]_{i}=\sum_{i=1}^{p}[\eta]_{i}=1$.

\section{B. Robust Control Invariant Set}

Consider the constrained discrete-time system

$$
\begin{aligned}
x(k+1)= & f_{d}(x(k), u(k), w(k)), \\
& x(k) \in \mathcal{X}, u(k) \in \mathcal{U} w(k) \in \mathcal{W},
\end{aligned}
$$

Definition 1: A set $\mathcal{C} \subseteq \mathcal{X}$ is a robust control invariant (RCI) set for (3) if $x \in \mathcal{C} \Rightarrow \exists u \in \mathcal{U}: f_{d}(x, u, w) \in$ $\mathcal{C}, \forall w \in \mathcal{W} . \mathcal{C}^{\infty} \subseteq \mathcal{X}$ is the maximal RCI (mRCI) set in $\mathcal{X}$, if it is RCI and contains all the other RCI sets in $\mathcal{X}$.

Given a RCI set $\mathcal{C}$ for (3), the set of admissible state-input pairs is $\mathcal{C}_{x, u}^{\infty}=\left\{x \in \mathcal{C}, u \in \mathcal{U}: f_{d}(x, u, w) \in \mathcal{C}, \forall w \in \mathcal{W}\right\}$. The set $\mathcal{C}_{x, u}^{\infty}$ is the set of admissible state-input pairs for $\mathcal{C}^{\infty}$.

The mRCI set construction relies on the Pre-set operator

$$
\begin{aligned}
& \operatorname{Pre}(\Omega, \mathcal{U}, \mathcal{W})= \\
& \quad\left\{x \in \mathcal{X}: \exists u \in \mathcal{U}, f_{d}(x, u, w) \subset \Omega, \forall w \in \mathcal{W}\right\},
\end{aligned}
$$

which computes the set of states that can be robustly driven to the target set $\Omega \in \mathbb{R}^{n}$ in one step. Based on (4), $\mathcal{C}^{\infty}$ for (3) is the fixpoint of the sequence $\left\{\Omega_{k}\right\}_{k}$, where $\Omega_{0}=\mathcal{X}$, $\Omega_{k}=\operatorname{Pre}\left(\Omega_{k-1}, \mathcal{U}, \mathcal{W}\right) \cap \Omega_{k-1}$. Indeed, $\Omega_{k} \subseteq \Omega_{k-1}$, and if there exists $\bar{k} \in \mathbb{Z}_{+}$such that $\Omega_{\bar{k}}=\Omega_{\bar{k}-1}, \Omega_{\bar{k}}=\mathcal{C}^{\infty}$. Conditions for convergence are discussed, e.g., in [11].

For the dpLDI system (1), the RCI set is $\mathcal{C}=\{x \in \mathcal{X}$ : $\left.\exists u \in \mathcal{U}, A_{i} x+B_{i} u+B_{w} w \in \mathcal{C}, \forall i=1, \ldots, \ell, \forall w \in \mathcal{W}\right\}$ and the Pre-set computation is detailed, e.g., in [10].

\section{Soft Landing Control}

Soft landing control aims at stopping a moving object in (and only in) a target region $\mathcal{E}_{\text {tgt }}$, while the object speed-ofapproach is kept below an upper bound that decreases as the distance from the target decreases [10]. The dynamics of the object moving in a one dimensional space is

$$
\ddot{d}(t)=\dot{v}(t)=\frac{1}{m} \sum_{i=1}^{n_{f}} F_{i}(t),
$$

where $d$ is the position with respect to the target, $v$ is the velocity, and $F_{i}, i=1, \ldots, n_{f}$ are the external forces. Here, we assume $d(0)<0, v(0)>0,0 \in \operatorname{int}\left(\mathcal{E}_{\text {tgt }}\right)$, where $\mathcal{E}_{\text {tgt }}=$ $\left\{d \in \mathbb{R}: \epsilon_{\min } \leq d \leq \epsilon_{\max }\right\}, \epsilon_{\min } \leq \epsilon_{\max }$.

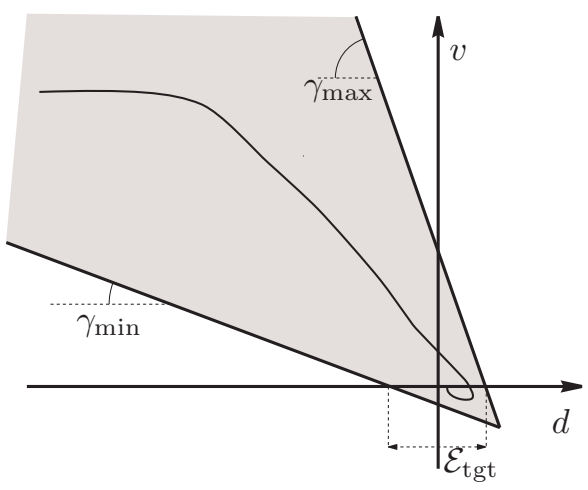

Fig. 1. Schematics of the soft landing problem

Result 1 ( [10]): Any infinite-time admissible trajectory of

$$
\begin{aligned}
\dot{x}(t)= & f(x(t), u(t), \vartheta(t)) \\
y(t)= & {[d(t) v(t)]^{T}=h(x(t)) } \\
& {[y(t)]_{2} \leq \gamma_{\max }\left(\epsilon_{\max }-[y(t)]_{1}\right) } \\
& {[y(t)]_{2} \geq \gamma_{\min }\left(\epsilon_{\min }-[y(t)]_{1}\right) } \\
& x(t) \in \overline{\mathcal{X}}, u(t) \in \mathcal{U} .
\end{aligned}
$$

where $x \in \overline{\mathcal{X}} \subseteq \mathbb{R}^{n_{x}}, u \in \mathcal{U} \subseteq \mathbb{R}^{n_{u}}, y=[d v]^{T} \in \mathcal{Y} \subseteq \mathbb{R}^{2}$, and $\vartheta \in \mathcal{O} \subseteq \mathbb{R}^{n_{\vartheta}}$ is the uncertainty, $\gamma_{\min }, \gamma_{\max } \in \mathbb{R}_{+}$, $\gamma_{\min }<\gamma_{\max }$, and (6e) are constraints on states and inputs solves the soft landing problem.

The constraints (6c), (6d) define the soft landing cone, see Figure 1. For computational purposes, in what follows (6a), (6b) are linear subject to additive disturbances and parametric uncertainty, and $\overline{\mathcal{X}}, \mathcal{U}$ are polytopes.

\section{Problem Statement}

Consider $\mathcal{P}_{1}=\left\{\theta \in \mathbb{R}^{\ell}: 0 \leq[\theta]_{i} \leq 1, \forall_{i=1}^{\ell}, \sum_{i=1}^{\ell}[\theta]_{i}=\right.$ $1\}, \mathcal{P}_{2}=\left\{\eta \in \mathbb{R}^{p}: 0 \leq[\eta]_{i} \leq 1, \forall_{i=1}^{p}, \sum_{i=1}^{p}[\eta]_{i}=1\right\}$ and for an appropriately small sampling period $T_{s}$, let (6a) be represented in discrete-time by

$$
x(k+1)=A_{s} x(k)+B_{s} u(k)+B_{w} w_{s},
$$

where $x \in \mathbb{R}^{n_{x}}, u \in \mathbb{R}^{n_{u}}, w_{s} \in \mathbb{R}^{n_{w}}, A_{s}=\sum_{i=1}^{\ell}[\theta]_{i} A_{i}$, $B_{s}=\sum_{i=1}^{\ell}[\theta]_{i} B_{i}, w_{s}=\sum_{i=1}^{p}[\eta]_{i} w_{i}$, and $B_{w}, A_{i}, B_{i}$, $i=1, \ldots, \ell$, and $w_{i}, i=1, \ldots, p$ are known, while $\theta \in \mathcal{P}_{1}$, $\eta \in \mathcal{P}_{2}$ are unknown.

Let $\mathcal{X}, \mathcal{U}$ be polyhedral sets whose elements satisfy (6c), (6d), (6e). Based on Sections II-A, II-B, we obtain the mRCI and the set of admissible state-input pairs

$$
\begin{aligned}
& \mathcal{C}^{\infty}=\left\{x \in \mathbb{R}^{n_{x}}: H^{\infty} x \leq K^{\infty}\right\}, \\
\mathcal{C}_{x, u}^{\infty}= & \left\{x \in \mathcal{C}^{\infty}, u \in \mathcal{U}: H_{x}^{\infty} x+H_{u}^{\infty} u \leq K_{u}^{\infty}\right\} .
\end{aligned}
$$

Let $\mathcal{P}=\mathcal{P}_{1} \times \mathcal{P}_{2} \subset \mathbb{R}^{\ell+p}$, and $\vartheta=\left[\theta^{T} \eta^{T}\right]^{T} \in \mathcal{P}$ denote the unknown parameters. For any $\bar{\vartheta}=\left[\begin{array}{ll}\bar{\theta}^{T} & \bar{\eta}^{T}\end{array}\right]^{T} \in \mathcal{P}$ we obtain the prediction model

$$
x_{k+1}=\bar{A} x_{k}+\bar{B} u_{k}+B_{w} \bar{w},
$$

where $\bar{A}=\sum_{i=1}^{\ell}[\bar{\theta}]_{i} A_{i}, \quad \bar{B}=\sum_{i=1}^{\ell}[\bar{\theta}]_{i} B_{i}$, and $\bar{w}=$ 
$\sum_{i=1}^{p}[\bar{\eta}]_{i} w_{i}$, and the trajectories of (9) are included in (1). Consider the MPC policy that at every step solves the finite horizon optimal control problem

$$
\begin{array}{cl}
\min _{U} & J^{c}=F\left(x_{N}\right)+\sum_{i=0}^{N-1} L\left(x_{i}, u_{i}\right) \\
\text { s.t. } & x_{i+1}=\bar{A} x_{i}+\bar{B} u_{i}+B_{w} \bar{w} \\
& u_{i} \in \mathcal{U} \\
& H_{x}^{\infty} x_{0}+H_{u}^{\infty} u_{0} \leq K_{u}^{\infty} \\
& x_{0}=x(k),
\end{array}
$$

where $U=\left[u_{0}, \ldots, u_{N-1}\right]^{T}, N \in \mathbb{Z}_{+}$is the prediction horizon, $F$ and $L$ are the terminal and stage cost, respectively, and applies $u(k)=u_{0}^{*}$, where $U^{*}=\left[u_{0}^{*}, \ldots, u_{N-1}^{*}\right]^{T}$ is the optimal solution of (10). By the properties of the RCI set, for any initial state $x(0) \in \mathcal{C}^{\infty},(10)$ is recursively feasible and hence the closed loop satisfies the constraints for all $k \in \mathbb{Z}_{+}$, for all admissible disturbances and parameter values.

Thus, the MPC policy based on (10) robustly enforces constraints on the dpLDI system (1), and hence by Result 1, it solves the soft landing control problem. However, the transient performance in terms of energy, time, jerk, i.e., the trajectory to achieve soft landing, is significantly affected by the uncertainty in the system parameters. Thus, here we aim at developing a control strategy with the following features.

Problem 1: Given $\left\{A_{i}, B_{i}\right\}_{i=1}^{\ell},\left\{w_{i}\right\}_{i=1}^{p}$ and $B_{w}$ for (7), design a robust dual control MPC (RDC-MPC) for soft landing of (7) that: $(i)$ guarantees $x(k)$ in $\mathcal{C}^{\infty}$, and hence $x(k) \in \mathcal{X}, u(k) \in \mathcal{U}$, for all $k \in \mathbb{Z}_{+},(i i)$ excites the system to improve learning of $\vartheta \in \mathcal{P}$ (iii) trades off excitation and control objectives based on the reliability of the estimated prediction model. Furthermore, $(i v)$ given a convex $J^{c}$, the RDC-MPC should only solve convex problems.

\section{Robust Dual Control MPC}

Next, we propose a two-steps RDC-MPC for uncertain systems modeled as dpLDI systems which combines the objectives of control and excitation for parameter estimation.

Definition 2: ( [8]) An input profile is persistently exciting if for every $k \in \mathbb{Z}_{0+}$ there exists $\Upsilon \in \mathbb{Z}_{+}$and $\rho_{0} \in \mathbb{R}_{+}$, such that parameter information matrix $R_{k}$ (the inverse of the covariance matrix) satisfies $R_{k+\Upsilon}-R_{k} \succeq \rho_{0} I$.

\section{A. Constrained RLS}

For system (7), we form a linear regression model for the unknown coefficients vector $\vartheta=\left[\begin{array}{ll}\theta^{T} & \eta^{T}\end{array}\right]^{T}$ given by

$$
\begin{aligned}
& x(k+1)+\epsilon(k+1)=\sum_{i=1}^{\ell}[\theta]_{i}\left(A_{i} x(k)+B_{i} u(k)\right) \\
& +\sum_{i=1}^{p}[\eta]_{i} B_{w} w_{i}+\epsilon(k+1)=M^{T}(k) \vartheta(k+1)+\epsilon(k+1),
\end{aligned}
$$

where $M(k)=\left[A_{1} x(k)+B_{1} u(k), \ldots, A_{\ell} x(k)+\right.$ $\left.B_{\ell} u(k), B_{w} w_{1}, \ldots, B_{w} w_{p}\right]^{T}$ is the regressor matrix, $\nu(k+$ $1)=x(k+1)+\epsilon(k+1)$, and $\epsilon(k+1)$ is measurement noise that is introduced for estimator design.
Recursive least squares (RLS) filters have been extensively used to implement dual control systems [3]-[6]. However, the parameter vector in (11) needs to be constrained in $\mathcal{P}$, which is needed to ensure that the corresponding prediction model generates trajectories included in those of (2), otherwise the guaranteed robust feasibility of the MPC optimal control problem (10). Thus, here we use a constrained RLS (cRLS)

$$
\begin{aligned}
K(k+1)= & P(k) M(k)\left(\alpha I+M^{T}(k) P(k) M(k)\right)^{-1} \\
\hat{\vartheta}(k+1)= & \underset{\vartheta \in \mathcal{P}}{\arg \min }\left\|\nu(k+1)-M^{T}(k) \vartheta\right\|^{2} \\
& +\|\hat{\vartheta}(k)-\vartheta\|_{\alpha R(k)}^{2} \\
P(k+1)= & \frac{1}{\alpha}\left(I-K(k+1) M^{T}(k)\right) P(k) \\
R(k+1)= & \alpha R(k)+M(k) M^{T}(k)
\end{aligned}
$$

where $P(k) \in \mathbb{R}^{(\ell+p) \times(\ell+p)}$ is the covariance matrix, $R(k) \in \mathbb{R}^{(\ell+p) \times(\ell+p)}$ is the information matrix, and $0<$ $\alpha \leq 1$ is the forgetting factor.

\section{B. Robust MPC with Active Learning}

We propose a two-steps RDC-MPC, which consists of an input design step and a control step with robust constraint guarantees such that it only solves convex problems.

For the input design step the minimum eigenvalue of the change in the information matrix along the input design horizon $\Upsilon, \lambda_{\min }\left(R_{\Upsilon}-R_{0}\right)$ is adopted as a as the measure of the excitation provided by an input sequence. As we will show later, with this choice we can obtain a convex relaxation that allows to design a RDC-MPC that only solves convex problems. We formulate the problem for the input design step as

$$
\begin{array}{cl}
\max _{U_{\text {exc }}(k)} & \lambda_{\min }\left(R_{\Upsilon}-R_{0}\right) \\
\text { s.t. } & x_{i+1}=\hat{A}_{k} x_{i}+\hat{B}_{k} u_{\text {exc } i}+B_{w} \hat{w}_{k} \\
& R_{i+1}=M_{i}\left(x_{i}, u_{\mathrm{exc}, i}\right) M_{i}^{T}\left(x_{i}, u_{\mathrm{exc}, i}\right)+\alpha R_{i} \\
& H_{x}^{\infty} x_{0}+H_{u}^{\infty} u_{\mathrm{exc}, 0} \leq K_{u}^{\infty} \\
& R_{0}=R(k),
\end{array}
$$

where the information matrix $R_{i}$ is computed from $x_{i}$ and $u_{i}$, and $U_{\text {exc }}(k)=\left[u_{\text {exc }, 1}, u_{\text {exc }, 2}, \cdots, u_{\text {exc }, \Upsilon}\right]^{T}$ is the input sequence designed to excite the system.

For the control step, similarly to [4]-[7], we add to the MPC cost function a term that favors the system excitation. Here, the additional term penalizes the deviation of the control input sequence from the input sequence designed to excite the system in a way that retains convexity

$$
\begin{array}{llr}
\min _{U} & J^{c} \\
& +\beta\left(x(k)-M^{T}(k-1) \hat{\vartheta}(k)\right)\left\|U-U_{\mathrm{exc}}(k)\right\|_{I}^{2}(14 \mathrm{~b}) \\
\text { s.t. } & x_{i+1}=\hat{A}_{k} x_{i}+\hat{B}_{k} u_{i}+B_{w} \hat{w}_{k}, \forall_{i=0}^{N-1} \\
& u_{i} \in \mathcal{U}, \forall_{i=0}^{N-1} \\
& H_{x}^{\infty} x_{0}+H_{u}^{\infty} u_{0} \leq K_{u}^{\infty}, \\
& x_{0}=x(k),
\end{array}
$$


where $\beta(\cdot)$ is a positive, non-decreasing function of the norm of the estimation error. When the prediction model represents well the system dynamics, the relative importance of the control objective increases, while when the prediction model does not represent well the system dynamics, more emphasis is placed on following the excitation input, which facilitates learning. The complete two-steps RDC-MPC framework is described in Algorithm 1.

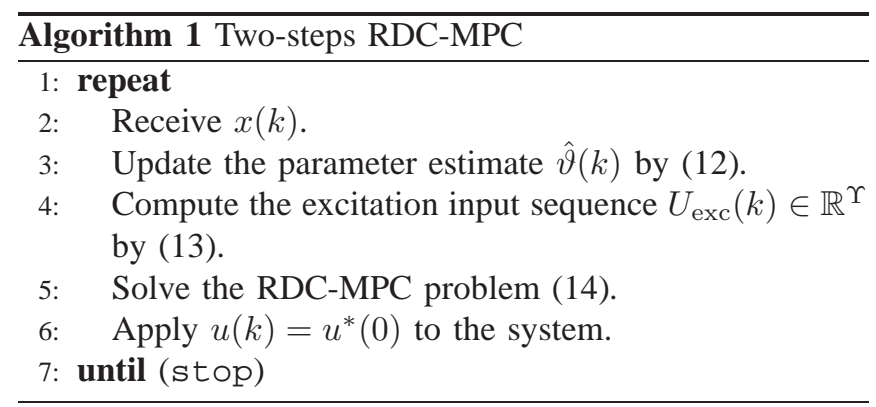

If $J_{c}$ is convex, (14) is a convex problem. However, (13) is still non-convex, which is addressed next.

\section{Convex Relaxation of InPut Design Problem}

In order to obtain an RDC-MPC algorithm that only solves convex problems, we solve (13) by a sequence of convex relaxations. The information matrix at step $i$ is

$$
R_{i}=\alpha^{i} R_{0}+\sum_{j=0}^{i-1} \alpha^{j} M_{i-j-1} M_{i-j-1}^{T} .
$$

From (15), each component of $R_{d}=R_{\Upsilon}-R_{0}$ is a quadratic function of $U,\left[R_{d}\right]_{i, j}=U^{T} Q_{i j} U+f_{i j}^{T} U+c_{i j}$. Furthermore, for $X$ positive semidefinite

$$
\begin{aligned}
& \lambda_{\min }(X)=\rho^{*}=\max \rho \\
& \text { s.t. } \quad X-\rho I \succeq 0
\end{aligned}
$$

which is a convex problem. Thus, we obtain the following.

Theorem 1: Problem (13) is equivalent to

$$
\begin{array}{ll}
\min _{\tilde{U}, U, \rho}-\rho \\
\text { s.t. } \quad R_{d}-\rho I \succeq 0 \\
\quad\left[R_{d}\right]_{i, j}=\operatorname{Tr}\left(Q_{i j} \tilde{U}\right)+f_{i j}^{T} U+c_{i j} \forall_{i, j=1}^{\ell+p} \\
\quad V=\left[\begin{array}{cc}
\tilde{U} & U \\
U^{T} & 1
\end{array}\right] \succeq 0 \\
\quad \operatorname{rank}(V)=1 \\
\quad A U-b \leq 0
\end{array}
$$

where (17f) models constraints (13b), (13d).

Proof (sketch): (Sketch) $\operatorname{rank}(V)=1$ if and only if there exists a vector $U$, such that $\tilde{U}=U U^{T}$. In turn, $\tilde{U}=U U^{T}$ implies that $\left[R_{d}\right]_{i, j}=\operatorname{Tr}\left(Q_{i j} \tilde{U}\right)+f_{i j}^{T} U+c_{i j}=$ $\operatorname{Tr}\left(Q_{i j} U U^{T}\right)+f_{i j}^{T} U+c_{i j}=U^{T} Q_{i j} U+f_{i j}^{T} U+c_{i j}$.

Problem (17) is convex except for the rank-1 constraint (17e). While there exists algorithms and tools for enforcing (17e) directly, see, e.g., [12], the computational burden increases prohibitively with the problem dimension. $\overline{\text { Algorithm } 2 \text { Relaxation of (17) using Rank Minimization }}$ and Bisection

1: $\left\{\tilde{U}^{*}, U^{*}, \rho^{*}\right\}=$

$$
\begin{array}{ll} 
& \arg \min _{\tilde{U}, U, \rho}-\rho, \\
\text { s.t. } \quad & R_{d}-\rho I \succeq 0, \\
& {\left[R_{d}\right]_{i, j}=\operatorname{Tr}\left(Q_{i j} \tilde{U}\right)} \\
& +f_{i j}^{T} U+c_{i j}, \quad \forall_{i, j=1}^{\ell+p} \\
& V=\left[\begin{array}{cc}
\tilde{U} & U \\
U^{T} & 1
\end{array}\right] \succeq 0, \\
& A U-b \leq 0,
\end{array}
$$

2: calculate upper and lower bounds for $\rho_{f}$,

$$
\begin{aligned}
& {[X]_{i, j}=\operatorname{Tr}\left(Q_{i j} U^{*} U^{* T}\right)+f_{i j}^{T} U^{*}+c_{i j}, \forall_{i, j=1}^{\ell+p},} \\
& \rho_{\max } \leftarrow \rho^{*}, \\
& \rho_{\min } \leftarrow \lambda_{\min }(X) .
\end{aligned}
$$

3: while $\left(\rho_{\max }-\rho_{\min }\right) / \rho_{\max }>\delta_{1}$ do

4: $\quad \rho_{f} \leftarrow 0.5\left(\rho_{\min }+\rho_{\max }\right), W^{(0)} \leftarrow I, h \leftarrow 0$

5: repeat

6: $\left\{\tilde{U}^{*}, U^{*}\right\}=$

$$
\arg \min _{\tilde{U}, U} \operatorname{Tr}\left(W^{(h)} V^{(h)}\right)
$$

$$
\text { s.t } \quad R_{d}-\rho_{f} I \succeq 0,
$$$$
\left[R_{d}\right]_{i, j}=\operatorname{Tr}\left(Q_{i j} \tilde{U}\right)+f_{i j}^{T} U+c_{i j},
$$$$
V^{(h)}=\left[\begin{array}{cc}
\tilde{U} & U \\
U^{T} & 1
\end{array}\right] \succeq 0,
$$$$
A U-b \leq 0
$$

7: $\quad W^{(h+1)} \leftarrow\left(\mathbf{V}^{(h)}+\sigma_{2}\left(\mathbf{V}^{(h)}\right) I\right)^{-1}$ where

$$
\mathbf{V}^{(h)}=\left[\begin{array}{cc}
\tilde{U}^{*} & U^{*} \\
U^{* T} & 1
\end{array}\right]
$$

and $h \leftarrow h+1$

8: until $\sigma_{2}\left(\mathbf{V}^{(h-1)}\right) \leq \delta_{2} \sigma_{1}\left(\mathbf{V}^{(h-1)}\right)$; or (18) is infeasible; or $h=h_{\max }$.

9: if $\sigma_{2}\left(\mathbf{V}^{(h-1)}\right) \leq \delta_{2} \sigma_{1}\left(\mathbf{V}^{(h-1)}\right)$ then

10: $\quad \rho_{\min } \leftarrow \rho_{f}$ (rank-1 solution has been found);

11: $\quad$ else

12: $\quad \rho_{\max } \leftarrow \rho_{f}$ (rank-1 solution has not been found).

13: end if

14: end while

Instead, here we exploit an inner-outer decomposition and a convex relaxation of the inner problem, which is described in Algorithm 2, where $\delta_{1}, \delta_{2} \in \mathbb{R}_{+}$, and $h_{\max } \in \mathbb{Z}_{+}$ are parameters that determine the accuracy of the results, and $\sigma_{i}(V)$ denotes the $i$-th singular value of $V$. After the initialization phase (Steps 1,2), Algorithm 2 solves for a fixed value of $\rho=\rho_{f}$ a convex relaxation of the rank1 problem, being an iterative rank minimization problem where, at every iteration, the weighted nuclear norm of $V$ is minimized (Step 6), and the weight is updated (Step 7). The 
outer problem updates the value of $\rho$ (Step 4) by bisection based on the results of the inner problem (Steps 10, 12).

A detailed discussion of the properties and convergence of the inner problem can be found in [13]. Due to the bisection search, Algorithm 2 converges to the optimal solution of (17) with precision due to $\delta_{1}, \delta_{2}$, and to the maximum iterations $h_{\max }$. For guaranteeing persistence of excitation, Algorithm 2 can terminate when a Rank-1 solution to (18) is found and $\rho_{\min }>0$. Stopping Algorithm 2 before that may compromise persistence of excitation, but constraint enforcement is still guaranteed by (14). Next, the properties of RDC-MPC algorithm are summarized.

Proposition 1: Algorithm 1, where (13) is solved by Algorithm 2, $x(0) \in \mathcal{C}^{\infty}$, and $J^{c}$ is convex, solves Problem 1 in the sense that: $(i) u(k) \in \mathcal{U}, x(k) \in \mathcal{C}^{\infty}$ and hence $x(k) \in \mathcal{X}$, for all $k \in \mathbb{Z}_{0+}$, (ii), the control input excites the system, (iii) the trade off between control and excitation is based on the reliability of the estimated prediction model, (iv) only convex problems are solved.

Proof: (Sketch) $(i)$ is guaranteed by using $\mathcal{C}_{x, u}^{\infty}$, and the recursive feasibility of (14) follows from (12) enforcing $\left\{\left(\hat{A}_{k}, \hat{B}_{k}\right)\right\} \in \operatorname{co}\left\{\left(A_{i}, B_{i}\right)\right\}_{i=1}^{\ell}, \hat{w}_{k} \in \operatorname{co}\left\{w_{i}\right\}_{i=1}^{p}$. (ii) and (iii) follow by using (13) and (14b) for designing the excitation input sequence and accounting for it in the optimal control problem weighted by $\beta$, which is a nondecreasing function of the observed prediction error. Finally, (iv) follows from Algorithm 1 solving (12) at Step 2, (13) at Step 3, and (14) at Step 4. Due to the definition of $\mathcal{P}$, (12) is a convex constrained quadratic program. By Algorithm 2, (13) is solved by a sequence of convex LMIs. By the assumption on $J^{c}$ and (14b), (14) is a convex optimization problem.

\section{A Case Study on Transportation Systems}

We consider a large transportation vehicle moving on a straight line that has to stop in $\mathcal{E}_{\text {tgt }}=\{d \in \mathbb{R}:-0.3 \leq d \leq$ $0.3\} \mathrm{m}$. The vehicle dynamics are described by

$$
\begin{aligned}
\dot{d}(t) & =v(t), \\
\dot{v}(t) & =\frac{k_{a}}{r m} \chi(t)-\frac{c_{0} \mu g}{m}-\frac{c_{1}}{m} v(t), \\
\dot{\chi}(t) & =-\frac{1}{\tau_{a}} \chi(t)+\frac{1}{\tau_{a}} u(t),
\end{aligned}
$$

where $d[\mathrm{~m}], v[\mathrm{~m} / \mathrm{s}]$, and $\chi$ are position, velocity, and traction actuator state, respectively, $m[\mathrm{~kg}]$ is the vehicle mass, $r[\mathrm{~m}]$ is the wheel radius, and $k_{a}[\mathrm{Nm}]$ is the traction actuator gain. The rolling resistance and the bearing friction coefficients are $c_{0}, c_{1}$, respectively. Model (19) is discretized with $T_{s}=$ $0.75 \mathrm{~s}$, with a constant state $\zeta(k)=c_{0} \mu g / m$ for all $k \in \mathbb{Z}_{0+}$. The upper states and inputs are constrained, $d \in[-200,1]$, $v \in[-5,30], \chi \in[-1,1], u \in[-1,1]$.

We consider (19) that is affected by uncertainties in mass and actuator time constant, $m \in\left[\left(1-\delta_{m}\right) \bar{m},\left(1+\delta_{m}\right) \bar{m}\right]$ and $\tau_{a} \in\left[\left(1-\delta_{\tau a}\right) \bar{\tau}_{a},\left(1+\delta_{\tau a}\right) \bar{\tau}_{a}\right]$, where $\delta_{m}=0.25$ and $\delta_{\tau a}=0.05$ define the relative uncertainties. We model the system as a dpLDI (2) with vertex systems $\left\{A_{i}, B_{i}\right\}_{i=1}^{\ell}$, $\ell=4$, and $p=0$, and we compute the corresponding $\mathrm{mRCI}$ set and the related admissible state-input pairs.

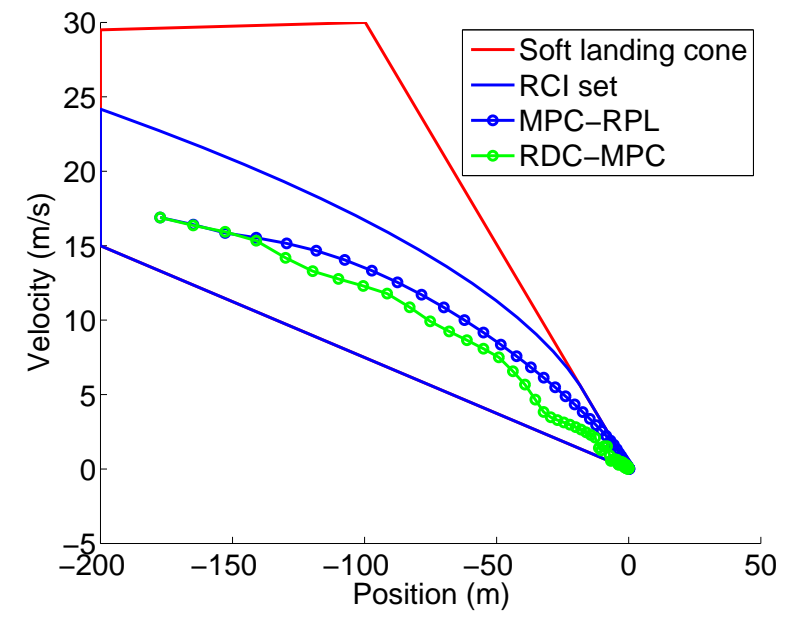

Fig. 2. Soft landing cone, RCI set, RDC-MPC and MPC-RPL trajectories

We develop a soft landing RDC-MPC and compare it with an MPC with RCI set with passive learning (MPC-RPL), i.e., the controller does not attempt to shape the input to increase the information on the system. The control stage cost function is $L(x, u)=x^{T} Q x+u^{T} R u+\Delta u^{T} R_{\Delta} \Delta u$, where $\Delta u(k)=u(k)-u(k-1)$ and the learning horizon $\Upsilon$ and the prediction horizon $N$ are equal, $\Upsilon=N=5$. We simulate the system in closed-loop with the RDC-MPC based on Algorithm 1, and MPC-RPL from the same initial conditions, which has been chosen randomly in a subset of the RCI set, far from the stopping range. In average Algorithm 2 solves 6 LMIs per iteration, each taking in average $0.15 \mathrm{~s}$.

Due to Result 1, both RDC-MPC and MPC-RPL stop the vehicle in the desired range, as shown in Figure 2. As shown by Figure 3, the RDC-MPC obtains a more significant reduction in the system uncertainty, as measured by the trace of the parameter estimation error covariance matrix, than MPC-RPL because it continues to excite the system. This results in the estimate converging close to their true values, as shown in Figure 4. As shown in Figure 5, RDC-MPC commands control inputs which provide higher excitation, without affecting constraint satisfaction or significantly sacrificing the closed-loop performance.

Next, we briefly show how RDC-MPC improves performance over the soft landing control method in [10] that does not learn the system parameters. For a slightly different cost function calibration, we compare an MPC with perfect model (MPC-PM), which represents the desired behavior, an MPC with uncertain model (MPC-UM), where both MPC-PM and MPC-UM are based on [10], and the RDC-MPC where the prediction model is learned during execution. Figure 6 shows a case where due to the error in the prediction model, MPC-UM operates close to the minimum velocity, which is significantly different from MPC-PM. Instead RDC-MPC learns the model parameters and produces a trajectory close to that of MPC-PM. 


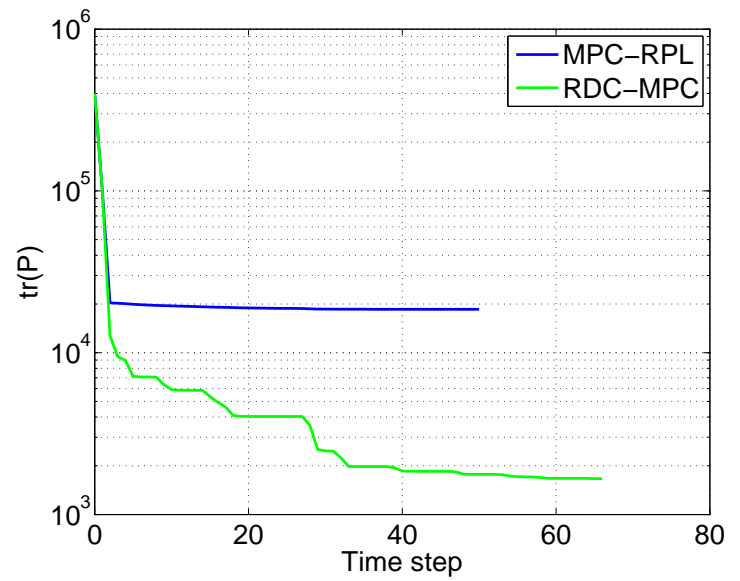

Fig. 3. Time histories of the trace of the predicted error covariance matrix
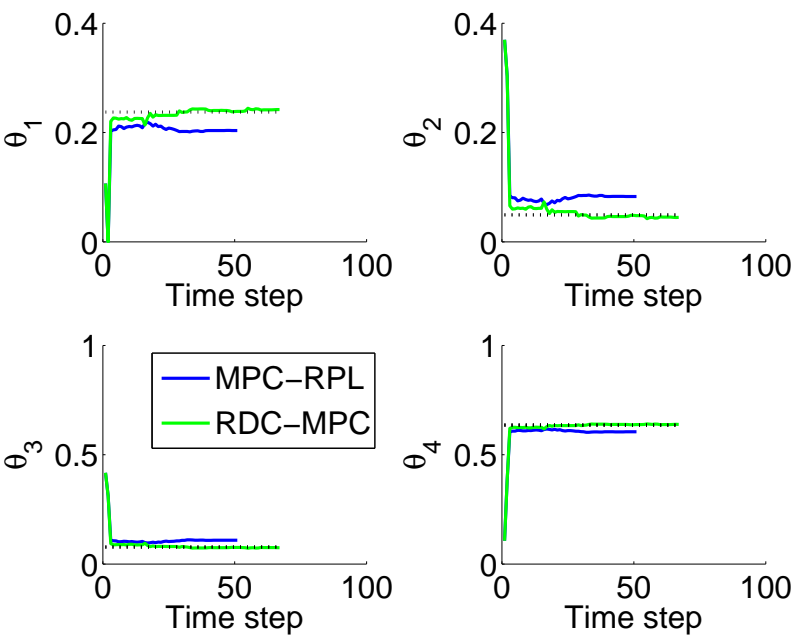

Fig. 4. Time histories of parameter estimates

\section{Conclusions}

We have developed a robust dual control MPC that simultaneously facilitates the estimation of the system parameters and controls the uncertain system. At every iteration first a feasible input sequence is designed to provide excitation, which is then used as a reference input in an MPC with RCI set constraints. The input design problem is solved by a rank minimization through a iteratively re-weighted nuclear norm minimization, so that the algorithm only solves convex problems. The method as been demonstrated on a soft landing control case study in transportation systems showing the advantages of RDC-MPC over passive learning, and the benefits of applying learning in the soft landing problem.

\section{REFERENCES}

[1] H. Genceli and M. Nikolaou, "New approach to constrained predictive control with simultaneous model identification," AIChE Journal, vol. 42, no. 10, pp. 2857-2868, 1996.

[2] M. S. Lobo and S. Boyd, "Policies for simultaneous estimation and optimization," in Proceedings of the American Control Conference, San Diego, CA, June 1999.

[3] G. Marafioti, R. Bitmead, and M. Hovd, "Persistently exciting model predictive control using fir models," in International Conference Cybernetics and Informatics, no. 2009, 2010, pp. 1-10.
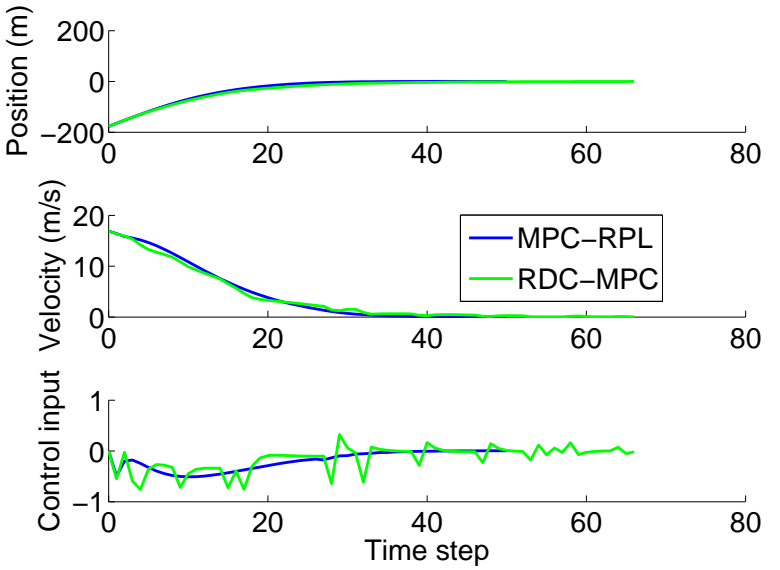

Fig. 5. Time histories of position, velocity, and input

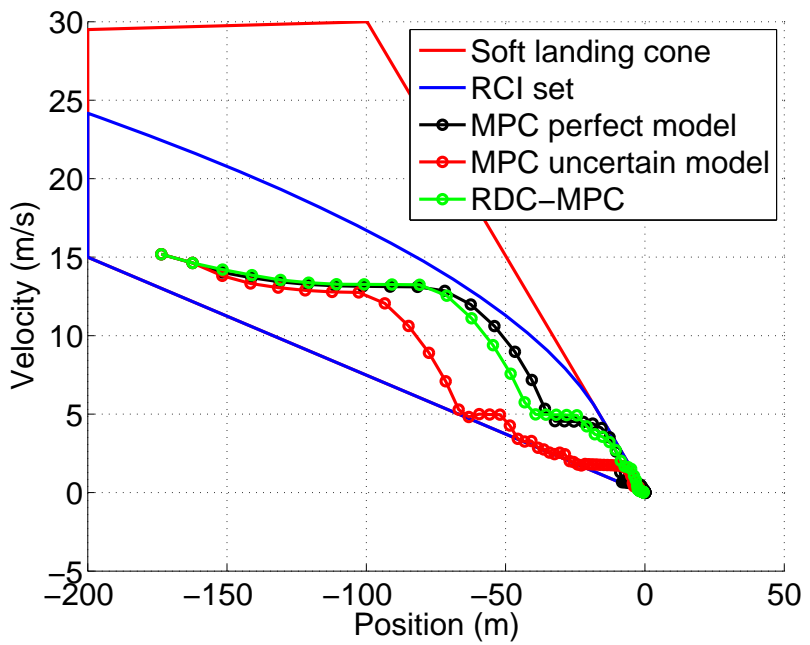

Fig. 6. Soft landing cone, RCI set, RDC-MPC, MPC-PM, MPC-UM trajectories

[4] J. Rathouskỳ and V. Havlena, "MPC-based approximate dual controller by information matrix maximization," International Journal of Adaptive Control and Signal Processing, vol. 27, no. 11, pp. 974-999, 2013.

[5] T. A. N. Heirung, B. E. Ydstie, and B. Foss, "An MPC approach to dual control," in 10th International Symposium on Dynamics and Control of Process Systems (DYCOPS)(Submitted), Mumbai, India, 2013.

[6] —-, "An adaptive model predictive dual controller," in Adaptation and Learning in Control and Signal Processing, vol. 11, no. 1, 2013, pp. 62-67.

[7] A. Weiss and S. Di Cairano, "Robust dual control MPC with guaranteed constraint satisfaction," in Proceedings of IEEE Conference on Decision and Control, Losa Angeles, CA, December 2014.

[8] G. C. Goodwin and K. S. Sin, Adaptive filtering prediction and control. Dover Publications, 2009.

[9] M. Tanaskovic, L. Fagiano, R. Smith, and M. Morari, "Adaptive receding horizon control for constrained mimo systems," Automatica, vol. 50, no. 12, pp. 3019-3029, 2014.

[10] S. Di Cairano, A. Ulusoy, and S. Haghighat, "Soft-landing control by control invariance and receding horizon control," in American Control Conference (ACC), 2014. IEEE, 2014, pp. 784-789.

[11] E. C. Kerrigan, "Robust constraint satisfaction: Invariant sets and predictive control," Ph.D. dissertation, $\mathrm{PhD}$ thesis, Cambridge, 2000.

[12] R. Orsi, U. Helmke, and J. B. Moore, "A Newton-like method for solving rank constrained linear matrix inequalities," Automatica, vol. 42, no. 11, pp. 1875-1882, November 2006.

[13] K. Mohan and M. Fazel, "Iterative reweighted algorithms for matrix rank minimization," The Journal of Machine Learning Research, vol. 13, no. 1, pp. 3441-3473, 2012. 